\title{
Tamizaje prenatal de enfermedades fetales genéticas y adquiridas mi- diendo tres marcadores en suero materno: determinación de AFP, BHcG y E3 no conjugados
}

\author{
Acuña J.M.*; de Elejalde M.M.**; Elejalde B.R.***; Isaza C.****
}

\begin{abstract}
RESUMEN: La determinación de fetos anormales se ha basado tradicionalmente en la selección de pacientes por edad materna o antecedentes personales y familiares, detectando sólo un pequeño número de fetos anormales. La introducción de la medición y estandarización de tres marcadores en suero materno (Alfafetoproteína, AFP, gonadotropina coriónica, BHcG, y estrioles no conjugados, uE3) ha probado ser una herramienta útil en la selección de un grupo importante $(54 \%$ a $67 \%)$ de pacientes que sin tener otra indicación para diagnóstico prenatal, gestan fetos anormales y/o tienen gestaciones anormales. La dificultad de la aplicación del triple marcador estriba en: 1. la necesidad de tener curvas estandarizadas para su interpretación, 2. la interpretación cuantitativa de los valores absolutos de la prueba basados en programas de computador complejos y costosos y 3. la interpretación clínica de los resultados basada en un análisis de regresión gaussiano de triple variable, haciendo que los valores obtenidos sean de utilidad al clínico.
\end{abstract}

La presente revisión proporciona las líneas de manejo, indicaciones y protocolos actuales de aplicación del tamizaje con Triple Marcador.

PALABRAS CLAVES: Triple marcador, alfafetoproteína, gonadotropina coriónica, astrioles no conjugados, diagnóstico prenatal.

SUMMARY: Determination of the abnormal fetuses has been done using maternal age and previous family history, detecting only a small percentage of abnormal fetuses. Introduction of biochemical maternal serum substances (AFP, BHcG, uE3), has provided a usefull tool to determine a high percentage $(54 \%$ to $67 \%$ ) of abnormal fetuses/gestations of those woman who do not have other indication to undergo complete prenatal diagnosis. So far, the limitations of this programs are the standarization fo the absolute values, the interpretation of the values with complex and expensive computer programs and the clinical interpretation of those values. This review gives the current application and concepts involved in the Triple Marker Screening.

KEY WORDS: Triple marker, alfafetoprotein, chorionic gonadotropin, unconjugates estriols, prenatal diagnosis.

\section{El problema}

La mayoría de las mujeres $(80 \%)$ cuyos fetos están afectados por trisomía 21 (causante del síndrome de Down) son menores de 35 años. La mayoría de los bebés con otras trisomías cromosómicas nacen del mismo grupo de mujeres. Además la mayoría de ellas no tienen historia familiar de esta condición, ni ninguna otra razón para pensar que necesitan diagnóstico genético prenatal. Tradicionalmente el diagnóstico genético prenatal se le ofrece a aquellas mujeres mayores de 35 años o aquellas que tienen historia familiar de síndrome de Down, otras anormalidades cromosómicas o defectos genéticos. Esto deja la mayoría (más de 90\%) de las familias que dan a luz niños anormales (afectados por enfermedades congénitas y heredadas) sin que se les ofrezca diagnóstico genético prenatal debido a la falta de indicaciones $(1,7)$.

\footnotetext{
* Profesor Asistente, Medicina, Universidad Nacional de Colombia.

** Directora del programa de Triple Marcador de Wisconsin, U.S.A.

*** Director Médico, Medical Genetics Institute, Wisconsin.

**** Profesor Asociado, Departamento de Morfología, Universidad del Valle.
}

\section{La necesidad}

Una vez que una mujer se embaraza, dará a luz un niño que puede ser normal o anormal. Aún antes de concebirlos, todos nuestros deseos, ilusiones y esperanzas de una vida mejor son para nuestros hijos. Aunque los avances de la medicina y la sociedad ayudan para que personas minusválidas puedan llevar vidas casi normales, individuos con capacidades físicas o mentales disminuidas tienen menos posibilidades de desarrollo personal. A consecuencia de esto sus logros serán menores que los de individuos normales. Algunos recién nacidos con enfermedades congénitas, heredadas o adquiridas graves son severamente inválidos y serán total o parcialmente dependientes de sus padres y de la sociedad durante toda su vida.

En una sociedad individualista y competitiva, la vida depende más en las habilidades individuales para sobrevivir. Las parejas que están planeando la concepción de sus hijos buscan consejo antes de la concepción para tratar de evitar aquellas circunstancias que pudiesen alterar el desarrollo normal del feto. Aunque es posible encontrar recomendaciones que sirvan, éstas son pocas. La mayoría de las anormali- 
dades fetales son debidas a causas que no podemos prevenir, modificar o prever. Muchas mujeres modifican sus actividades cotidianas con la esperanza de que un estilo de vida «más saludable» les asegurará tener un niño sano. La mayoría de las anormalidades que afectan el feto humano no se pueden prevenir con modificaciones en el estilo de vida.

Las parejas que están planeando tener un hijo no piensan que éste pueda ser anormal, excepto que hallen circunstancias que lo predispongan. En realidad cada embarazo está a riesgo de producir un niño anormal. El que los padres deseen tener un hijo normal y la falta de signos externos de anormalidad que sean reconocibles, crean la necesidad de un sistema para seleccionar las mujeres que pudiesen tener un riesgo elevado de tener un hijo anormal.

\section{Tamizaje}

Un examen de tamizaje debe poder seleccionar entre la población total de mujeres embarazadas a aquellas mujeres con riesgo elevado de dar a luz un hijo anormal, para que ellas tengan la posibilidad de que se les ofrezca un examen diagnóstico más específico. Durante un largo tiempo se ha sentido la necesidad de un examen de tamizaje para malformaciones congénitas, síndromes y defectos cromosómicos que sea de precio accesible y además confiable.

\section{El derecho a saber}

Las familias quieren la oportunidad y tienen el derecho a preguntar sobre la salud de el feto que han concebido (1). $\mathrm{Si}$ se prueba que el feto está sano el embarazo será mejor disfrutado; si el feto es anormal la familia tendrá varias opciones que puede considerar para afrontar o prevenir las consecuencias de esta anormalidad.

\section{Métodos usados anteriormente para ofrecer solucio- nes al problema}

\section{Alfafetoproteína}

La determinación de alfafetoproteína (AFP) en el suero materno se introdujo y probó ser una herramienta útil en la selección de mujeres con riesgo alto de tener fetos afectados por defectos del tubo neural y otros defectos caracterizados por aperturas de el cuerpo fetal. Fue usada durante muchos años con este propósito y se ha convertido en un examen mucho más importante al reconocerse que no sólo niveles altos de AFP en suero materno son un indicio de enfermedad fetal, sino que niveles bajos son también indicio de un riesgo elevado de enfermedad fetal (genética y adquirida). La medición del nivel de AFP en el suero materno ha sido usada durante varios años y es considerada parte del estándar de cuidado que debe ser ofrecido a toda mujer embarazada.

Si la alfafetoproteína en suero materno (AFPSM) es anormalmente alta (por encima de 2.0 múltiplos de la mediana (MoM) para algunos laboratorios y $2.5 \mathrm{MoM}$ para otros la paciente tiene un alto riesgo de:

1) Malformaciones que producen aperturas en el cuerpo fetal (defectos del tubo neural, gastrosquisis y onfalocele)

2) Prematurez y bajo peso al nacer
3) Gestación múltiple

4) Hemorragia materno-fetal

5) Hidrops fetal

6) Muerte fetal. Este grupo tiene un interés especial debido a que aquellas mujeres que tienen AFPSM elevada de origen no fetal (AFP en líquido amniótico normal) tienen un riesgo elevado de muerte fetal (para AFPSM $>3.0 \mathrm{MoM}$, tasa de riesgo es 10.4 veces más alta que la de aquellas mujeres que tienen AFPSM entre 0.5 y $1.9 \mathrm{MoM},(2)$.

7) Tumores maternos

8) Pre-eclampsia

9) Infecciones feto-placentarias

Cuando una paciente tiene un examen de tamizaje AFPSM positivo se le deben ofrecer exámenes diagnósticos (ultrasonido y amniocentesis). Los exámenes de tamizaje seleccionan individuos con riesgo elevado pero no son diagnósticos. Tampoco responden una pregunta; mejor, hacen una pregunta más específica. Aquellas mujeres que se someten a un examen diagnóstico tendrán uno de estos dos resultados:

1) El feto tiene uno de los defectos o enfermedades asociadas con AFPSM anormalmente baja o alta, o

2) El feto no tiene uno de estos defectos.

Repetidamente se ha demostrado que el segundo grupo de mujeres tiene un riesgo más elevado de complicaciones tardías del embarazo que las mujeres con AFPSM normal. Esto es aún más importante cuando la AFP en líquido amniótico (AFPLA) es normal y la AFPSM persiste elevada. Comúnmente esto se debe a infecciones fetales y placentarias o a fallo placentario, lo que indica que el embarazo es de alto riesgo (4).

La medición de AFPSM se ha convertido en uno de los elementos más poderosos en la determinación de la salud fetal y en la clasificación de los riesgos del embarazo. Recientemente la combinación con otros, $\beta$-Gonadotrofina coriónica humana en suero materno (BGChSM) y estrioles no conjugados en suero materno (uE3SM), ha aumentado el número de casos de síndrome de Down y otras anormalidades cromosómicas detectadas de $20 \%$ (sólo edad materna) a $40 \%$ (edad y AFPSM) a $61 \%$ (edad, AFPSM, BGChSM y uE3SM con corte en 1:190, tasa de falsos positivos 5\%) ó 67\% (edad, AFPSM, BGChSM y uE3SM con corte en $1: 270$, tasa de falsos positivos $7.2 \%$ ) (3).

\section{El nuevo método}

El programa de tamizaje usando la determinación de tres marcadores bioquímicos en suero materno

La información anterior es evidencia convincente de la importancia de la determinación de AFPSM como parte del cuidado obstétrico de rutina dado a toda mujer embarazada. AFPSM es la única forma disponible de identificar y reclasificar aquellas mujeres consideradas previamente como de bajo riesgo y que en realidad tienen un riesgo elevado de complicaciones severas del embarazo. Cuando la AFPSM se encuentra entre 2.5 y $2.9 \mathrm{MoM}, 18.5 \%$ de los embarazos tienen un resultado anormal, si la AFPSM es 3.0 a $4.9 \mathrm{MoM}$ este número se eleva a $28.5 \%$ y cuando está por encima de 5.0 MoM, $70.3 \%$ de los embarazos tienen un resultado anormal (4). 
Se ha encontrado que un nivel alto de $B G C h S M>1.5$ MoM se asocia con anormalidades cromosómicas, excepto trisomía 18 , monosomía $\mathrm{X}$ sin hidrops y triploidía, los cuales tienen un nivel bajo (5).

Canick demostró que las mujeres cuyos fetos tienen una anormalidad cromosómica (incluyendo síndrome de Down) tienen un nivel de uE3SM más bajo de lo normal (6).

\section{Medianas (Múltiplos de la mediana, MoM)}

La interpretación de los exámenes depende de la determinación de medianas para cada edad gestacional. La mediana es una medida estadística reproducible y fácil de obtener. El valor encontrado en cada paciente se expresa en medianas dividiendo la concentración encontrada por la mediana correspondiente a la semana de gestación (comúnmente determinada contando las semanas desde el primer día de la última regla y redondeando a la última semana completa).

\section{Tamaño fetal y edad gestacional discordantes}

Si los resultados son anormalmente bajos, se debe hacer un ultrasonido para determinar el tamaño fetal. Si el tamaño fetal está dentro del rango normal para edad gestacional estimada, o si la edad gestacional determinada por ultrasonido tiene menos de 15 días de diferencia con la edad gestacional determinada por la fecha de la última regla (FUR), no se hace ninguna corrección (3).

Si el tamaño del feto es mayor o menor de lo anterior, para los cálculos se usa la mediana específica para el diámetro biparietal (BPD) en vez de la mediana para la semana de gestación.

\section{Correcciones por raza, peso, diabetes y embarazo múltiple}

Los múltiplos de la mediana se deben corregir por raza (las mujeres de raza negra tienen AFPSM 10 a $15 \%$ más elevada que las mujeres de raza blanca), por peso (a mayor peso es más baja la mediana), diabetes (mujeres diabéticas tienen valores mayores que las mujeres no diabéticas), por embarazo múltiple (la concentración de AFPSM en mujeres con embarazos gemelares es el doble que la de mujeres con embarazos únicos).

A la paciente se le deben ofrecer exámenes diagnósticos prenatales si luego de estas correcciones el MoM es anormalmente alto o bajo (7-8).

La necesidad de corregir AFPSM por raza, peso, diabetes y embarazo múltiple ha sido claramente demostrada en la literatura. A pesar de que los datos para el ajuste de los MoM de BGChSM y uE3SM no son tan extensos, se ha encontrado que es necesario hacer correcciones similares a las descritas para AFPSM (9-10).

\section{Estudios confirmatorios}

El uso de programas de tamizaje usando la determinación de tres marcadores bioquímicos en suero materno ha sido estudiado, validado y encontrado de gran utilidad por grupos independientes en Europa y en los Estados Unidos. Estos programas constituyen uno de los avances más importantes en genética, cuidados prenatales, obstetricia y perinatología.
Un trabajo publicado recientemente confirma y convalida los resultados de reportes previos (11).

\section{Condiciones asociadas con resultados anormales de los tres marcadores}

La siguiente es una lista corta de las principales condiciones asociadas con resultados anormales en el examen de tamizaje con tres marcadores (Tabla 1).

Tabla 1

\begin{tabular}{|l|c|c|c|c|}
\hline Condición & AFPSM & BGChSM & uE3SM & Nota \\
\hline Síndrome de Down & Baja & Alta & Baja & \\
Monosomía X con hidrops & Baja/Normal & Alta & Baja & \\
Monosomía X sin hidrops & Baja/Normal & Baja & Baja & \\
Trisomfa 18 con defectos abiertos & Alta & Baja & Baja & \\
Trisomfa 18 sin defectos abiertos & Baja & Baja & Baja & \\
Triploidía & Baja/Normal & Baja & Baja & \\
Anencefalia & Alta & Baja/Normal & Baja & 1 \\
Espina bffida & Alta & Normal & Normal & \\
Gastrosquisis & Alta & Normal & Normal & \\
Onfalocele & Alta & Normal & Normal & \\
Hidrops & Normal & Alta & Baja & \\
Hidrops & Alta & $?$ & $?$ & \\
Nefrosis (tipo Finlandés) & Alta & Normal & Normal & \\
Oligohidramnios & Alta & $?$ & $?$ & \\
Polihidramnios & Baja & Normal & $?$ & \\
Infección fetal & Alta & $?$ & $?$ & 2 \\
Mole hidatiforme & Very low & Very high & $?$ & 1 \\
Muerte fetal reciente & Alta & Baja & Baja & 1 \\
Muerte intrauterina futura & Alta & Alta & $?$ & 3 \\
Prematurez & Alta & Alta & $?$ & 4 \\
Peso bajo al nacer & Alta & Alta & $?$ & 4 \\
Hipertensión materna & Alta & Alta & $?$ & 4 \\
\hline \multicolumn{2}{|c|}{ Baja AFPSM: 0.75 MoM } & Alta AFPSM: & 2.5 MoM & \\
Baja BGChSM: 0.55 MoM & Alta BGChSM: 2.0 MoM &
\end{tabular}

Baja uE3: $\quad 0.70 \mathrm{MoM}$

Notas: 1 . Se eleva hasta que alcanza una meseta a un nivel muy elevado.

2. Se eleva luego del inicio de la infección y disminuye a medida que la infección se cura.

3. Si el feto no tiene uno de los defectos que pueden producir AFPSM alta. Entre más alta la AFPSM, más alto el riesgo de muerte fetal.

4. Si el feto no tiene uno de los defectos que pueden producir AFPSM alta.

\section{Estimación, cálculo de riesgos y base de datos norma- tivos}

Una de las partes más complejas e importantes del programa de tamizaje con tres marcadores es el análisis matemático. Hay dos métodos disponibles:

1) El cálculo de tasas de probabilidad para cada marcador (1, 2 ó 3 de ellos) y luego multiplicar cada uno de los resultados individuales. Este método es incorrecto puesto que los tres marcadores están mutuamente correlacionados (11).

2) El cálculo de tasa de probabilidad (factor de ajuste del riesgo) basada en el método Gaussiano trivariable con la prueba de Shapiro-Wilk W-test. Este método es el más difícil y es el método correcto para el cálculo de riesgos (12-13). 
Para el cálculo de riesgos de síndrome de Down, anormalidades cromosómicas, defectos del tubo neural y otros resultados anormales del embarazo, nosotros usamos datos publicados para las tasas de riesgo (riesgo por edad materna). La tasa de probabilidad (factor de ajuste del riesgo) se calculan usando ecuaciones (para el análisis univariable y multivariable) y datos publicados por Wald NJ, Cuckle HS, Densem JW, et al., (13). Estas ecuaciones y datos son ampliamente usadas en todo el mundo. El programa de computador usado en el cálculo de estos riesgos emplea estos métodos matemáticos.

Los datos normativos de las medianas para diferentes edades gestacionales se obtuvieron en nuestro laboratorio, nosotros también utilizamos ecuaciones publicadas por los autores previamente mencionados. Los factores de corrección por raza, peso, diabetes y embarazo gemelar, son datos y ecuaciones tomados de la literatura. El uso de los datos y ecuaciones comúnmente aceptados hace que nuestros resultados de el programa de tamizaje con tres marcadores sean similares a los resultados de los exámenes hechos en otros laboratorios.

Se ha demostrado que los tres marcadores y los mecanismos de su variabilidad (desconocidos hasta el momento) son variables independientes con una ligera correlación por parejas (11).

Efecto de la elección del tipo de análisis matemático:

El efecto de la diferencia entre los métodos utilizados en el cálculo de el factor de ajuste del riego se vé claramente en el siguiente ejemplo:

Mujer blanca, 28 años de edad y 17 semanas de gestación, quien tiene una AFPSM: $0.53 \mathrm{MoM}$, BGChSM: $1.58 \mathrm{MoM}$, uE3SM: $0.73 \mathrm{MoM}$, un riesgo por edad de 1:890 para síndrome de Down. Cuando el cálculo se hace con el algoritmo Gaussiano multivariable el riesgo ajustado es 1:200; si el cálculo se hace obteniendo las tasas de probabilidad de cada marcador y multiplicando cada una de ellas se obtiene un riesgo de 1:276. El segundo método no tiene en cuenta la correlación que exista entre los marcadores y en este ejemplo esto causa una subestimación de el riesgo, 1:276 lo cual es incorrecto (14).

El uso del método incorrecto puede cambiar el resultado del examen de tamizaje de un examen positivo 1:200 a un examen negativo 1:276. En el primer caso la paciente será aconsejada para que se haga exámenes diagnósticos puesto que el examen de tamizaje es positivo. En el segundo caso la paciente será aconsejada debido a que el examen de tamizaje es negativo exámenes diagnósticos no están indicados.

Ofreciendo el examen: revelar las implicaciones que tiene el ser examinada y obtener permiso informado para hacer el examen

La paciente tiene el derecho de ser examinada. Para que esto sea un derecho y una opción la paciente debe tener también el derecho de no hacerse el examen. Esta debe ser una decisión informada tomada luego de que el médico cumpla su deber de revelar completamente la naturaleza e implicaciones de el examen de tamizaje usando tres marcadores. La paciente debe ser informada de que el examen:

1) Es para tamizaje de la población con el fin de seleccionar aquellas mujeres que tienen un riesgo elevado de entre las que tienen un riesgo normal o disminuido.
2) Si el examen es positivo no es diagnóstico, sino que indica que el riego es más elevado, lo que abre una incógnita y crea ansiedad porque hace más real la posibilidad de que el feto sea anormal.

3) $\mathrm{Si}$ el examen es negativo puede producir un falso sentido de confirmación de que el feto es normal, muchos embarazos anormales tienen resultados normales para los tres marcadores usados en este examen.

4) El examen se ofrece para darle a la familia la oportunidad de buscar exámenes diagnósticos en caso de que sea positivo.

5) Cuando el examen es negativo no debe interpretarse como una indicación de que el riesgo es más bajo de el riesgo por edad.

6) Sólo se debe hacer si la familia desea saber sobre la salud fetal.

Luego de que ha sido completamente informada de la naturaleza e implicaciones del examen, la familia tiene el derecho de aceptar o rechazarlo.

\section{Permiso informado}

El profesional de la salud tiene el deber de obtener permiso informado para hacer todo procedimiento, intervención o examen de laboratorio a un paciente. Entre los médicos no existe un consenso sobre la idea y el proceso específico que debe ser seguido para obtener permiso informado en el consultorio. Muchas personas creen en y practican permiso verbal, y normalmente lo documentan con una anotación en la historia clínica del paciente. Otros le suministran al paciente información escrita y le piden que firme una forma de permiso informado.

\section{Revelando los resultados de el examen, aconsejando}

Uno de los componentes más importantes dẹ el programa de tamizaje con tres marcadores es el revelar los resultados del examen y el consejo de obtener diagnóstico prenatal. La forma como se ofrece el tamizaje determina en gran parte los efectos que produce tanto cuando es positivo como negativo. Esto debe ser hecho por un individuo adecuadamente entrenado, suministrándole a la paciente toda la información sobre las implicaciones de los exámenes de tamizaje y diagnóstico, y debe tener un énfasis especial en responder todas las preguntas que la paciente pueda tener. En muchos casos las habilidades de un consejero genético son necesarias.

El proveer al paciente con información escrita y obtener permiso por escrito además de proveer información que la paciente puede consultar en busca de respuestas, evita en gran medida la angustia que se crea cuando el tamizaje es positivo y la paciente reacciona ante las malas noticias.

\section{Examen de tamizaje positivo}

Aproximadamente $6.6 \%$ de las pacientes que se someten a este examen tienen un resultado positivo. Algunos de estos son debidos a que la gestación es más temprana de lo estimado por la FUR. Luego de que la edad gestacional es corregida por ultrasonido $3.8 \%$ tienen un examen de tamizaje positivo para síndrome de Down; 58 to $60 \%$ de los fetos con síndrome de Down se encuentran en este grupo de mujeres. 
El examen de tamizaje puede producir también otros resultados positivos. Si la AFPSM es anormalmente alta (sobre 2.0 o $2.5 \mathrm{MoM}$, dependiendo del laboratorio), sugiere la presencia de condiciones tales como defectos del tubo neural, onfalocele, gastrosquisis, muerte fetal in utero, retardo del crecimiento intrauterino y otros resultados anormales del embarazo. Si los tres marcadores son anormalmente bajos el tamizaje es positivo para trisomía 18.

\section{Exámenes diagnósticos}

A todas las pacientes con examen de tamizaje positivo se les deben ofrecer exámenes diagnósticos. Si los resultados de los exámenes diagnósticos son positivos (anormales) esto explica el resultado positivo de el examen de tamizaje. Si el examen diagnóstico no revela ninguna anormalidad y la AFPSM o la BGChSM o ambas están elévadas, se considera que la paciente tiene un alto riesgo de tener aquellas complicaciones asociadas con AFPSM y BGChSM anormalmente altas. Esto es de gran ayuda puesto que permite identificar aquellas pacientes que necesitan seguimiento más cercano y mayor atención obstétrica.

\section{Ultrasonido y amniocentesis}

Estos son los exámenes más utilizados en diagnóstico prenatal. El ultrasonido se utiliza en todo momento durante el embarazo desde la cuarta semana de gestación hasta el momento del parto. Este método permite un análisis fidedigno y cuidadoso de las estructuras anatómicas, función, desarrollo y crecimiento del feto, placenta, tejidos y órganos abdominales maternos. Defectos del tubo neural y otros que producen aperturas del cuerpo fetal pueden ser diagnosticados siempre y cuando sean lo suficientemente grandes y el feto esté en una posición que permita observar la región afectada. Cuando se encuentran una o más malformaciones en un feto se debe sospechar que este feto está afectado por una anormalidad cromosómica.

Hay varios tipos de examen fetal por ultrasonido. Cuando se tiene un examen de tamizaje positivo, ultrasonido de alta resolución es el único tipo que se debe considerar. Cuando el médico consultante selecciona un consultor para hacer el examen por ultrasonido, debe considerar cuidadosamente sus habilidades como sonografista y especialmente sus habilidades diagnósticas y conocimiento de enfermedades fetales genéticas y adquiridas.

\section{Exámenes citogenéticos, bioquímicos y de biología molecular}

La selección y función de los métodos para obtener muestras para estudio de cromosomas fetales es diferente dependiendo de la edad gestacional. Biopsia de vellosidades cariónicas se puede hacer desde la octava semana, comúnmente luego de la décima semana. Normalmente la amniocentesis se hace luego de la semana 16 pero se ha demostrado que es igualmente segura y confiable cuando se hace desde la semana 12 y se puede hacer exitosamente desde la semana 10. Si la edad gestacional es muy cercana a la fecha límite para terminación del embarazo, el hacer una toma de sangre umbilical fetal por vía percutánea (PUBS) o una biopsia de placenta, permite que la familia tenga resultados del cariotipo fetal rápidamente cuando todas las posibilidades están aún disponibles. Cuando seleccione al médico consultor para estos procedimientos, debe considerar la experiencia de el individuo que va a hacer el examen, idealmente debe ser la misma persona que hace el ultrasonido. Su tasa de complicaciones y la disponibilidad de un laboratorio de alta calidad que pueda manejar tanto los procedimientos más simples como los más complejos son aspectos que deben jugar un papel muy importante en la decisión de escoger sus consultores clínicos y de laboratorio.

El feto o recién nacido deben ser examinados al nacimiento para corroborar los hallazgos de el ultrasonido y los exámenes citogenéticos, bioquímicos o de biología molecular.

\section{Beneficios}

De acuerdo con lo anterior, el examen de tamizaje con tres marcadores; la herramienta más útil en obstetricia y genética de la reproducción para la evaluación de los riesgos de resultados anormales del embarazo, y la selección de aquellas mujeres cuyo riesgo es alto, para hacerles un seguimiento más cercano y planear el manejo que pudiese prevenir complicaciones o reducir sus consecuencias.

\section{Requisitos}

Para que el programa de tamizaje con tres marcadores dé los mejores resultados, éste se debe hacer dentro de un programa comprensivo de genética médica y diagnóstico genético prenatal que provea apoyo en la interpretación de los resultados de los exámenes y servicios de seguimiento. Este programa debe además incluir servicios de diagnóstico y consejería para los pacientes que tengan resultados anormales. Cuando este examen se hace sin ser parte de un programa de este tipo, se convierte en un problema para los pacientes y los médicos.

\section{Limitaciones}

El examen de tamizaje con tres marcadores no es un examen diagnóstico. Los exámenes de tamizaje crean incógnitas, no las resuelven. En este caso un tamizaje positivo llama la atención al hecho de que la paciente tiene un riesgo mayor de lo normal. Los exámenes diagnósticos responden preguntas con exactitud; luego de someterse a ellos la paciente sabrá si el feto está o no está afectado por uno de los defectos asociados con un examen de tamizaje positivo.

\section{Riesgos del examen}

Quizás los riesgos más importantes del examen son las consecuencias psicológicas. Aquellas mujeres que tengan resultados negativos se sentirán seguras de la salud de sus fetos. Aquellas con resultados positivos sentirán la ansiedad generada por la posibilidad de que sus fetos sean anormales. Para reducir el sufrimiento de la familia el examen de tamizaje se debe hacer dentro de un sistema que pueda proveer exámenes diagnósticos de inmediato, con resultados en el menor tiempo posible.

La mayoría de las familias no consideran la posibilidad de que puedan tener un hijo anormal y cuando reciben un resultado de tamizaje positivo reaccionan con angustia, frus- 
tración y temor (de diversos grados de intensidad). Algunas de estas familias reaccionan con agresividad contra los individuos que hicieron el examen. Esto es entendible y requiere de cuidadoso consejo e intervención, puesto que esta reacción es una complicación médica creada al poner en duda la integridad de la salud del feto. El creer que el feto es normal es de gran importancia para la familia en estos momentos en los que la mayoría de la gente no está preparada para pensar que el feto pueda ser anormal y por lo tanto su potencial para el futuro pueda ser limitado. El revelar completamente los propósitos y consecuencias del examen y dar a la paciente la opción de aceptar o rechazar el examen previenen la mayoría de las reacciones severas.

Oțro riesgo importante del examen, es originado por los cálculos matemáticos del riesgo. Si el método utilizado no es el algoritmo Gaussiano multivariable, el riesgo puede ser sub o sobreestimado. Por lo tanto en algunos casos en los que no se debería hacer diagnóstico genético prenatal éste se hará, y en otros en los que se debería haber ofrecido diagnóstico prenatal éste no lo es. Este último grupo puede producir niños anormales que podrían haber sido detectados si se hubiese utilizado el método correcto.

Si en lugar de los tres marcadores se utilizan sólo dos, el riesgo será subestimado de forma significativa, y muchos casos de anormalidades fetales no serán detectados. Esta subestimación es aún mayor si se usan menos de tres marcadores y los riesgos se calculan con el análisis univariable en lugar del multivariable (trivariable).

Estos pueden ser prevenidos en gran medida, si se escoge el programa correcto para hacer el examen y si a la paciente se le suministra información clara y completa antes de tomar la muestra para el examen.

\section{Consecuencias}

El examen de tamizaje con tres marcadores permite que las familias que tienen un tamizaje positivo puedan buscar procedimientos diagnósticos para determinar antes del nacimiento si el feto es normal o anormal. Cuando esta información es usada de forma adecuada y sabia, ayuda a solucionar problemas humanos, algunos de los cuales son de por vida.

Cualquier programa de tamizaje debe cumplir cuidadosamente con los requisitos implícitos en la relación médicopaciente:

1) Documentar con examen

2) Revelar completamente lo encontrado y sus implicaciones

3) Dar recomendaciones para continuar el estudio del problema

4) Explicar los procedimientos alternativos

5) Obtener consentimiento informado

\section{Cómo seleccionar a dónde enviar el examen}

El laboratorio donde usted debe enviar sus muestras para el examen debe cumplir con los siguientes requisitos.

1) Hacer el análisis de los tres marcadores.

2) El examen se debe hacer en duplicado

3) Debe tener programas de control de calidad internos y externos de acuerdo con las regulaciones existentes.

4) Debe tener datos normativos para las semanas de gestación para las que se ofrece al examen.

5) Debe corregir los MoMs de los marcadores por peso, raza, diabetes y mellizos.

6) Debe proveer determinación del riesgo de defectos que producen apertura en el cuerpo fetal.

7) La determinación del riesgo debe hacerse usando el algoritmo Gaussiano multivariable (trivariable).

8) Debe producir reportes con interpretación completa y consejo de cómo proceder cuando el tamizaje es positivo.

9) Debe proveer explicaciones y consejería para los pacientes y médicos.

10) Debe tener un sistema completo de consejería genética y diagnóstico prenatal que responda las preguntas creadas por el examen de tamizaje positivo.

\section{Indicaciones:}

El examen está indicado en toda paciente embarazada, especialmente aquellas pacientes menores de 35 años.

\section{Abuso del examen:}

Este examen no debe reemplazar el estándar de cuidado para mujeres quienes tienen indicaciones para diagnóstico genético prenatal por su edad, historia familiar, hijos anormales, abortos múltiples, exposición a teratógenos o anormalidades del embarazo, incluyendo hallazgos ultrasonográficos anormales. El examen de tamizaje con tres marcadores es un examen de tamizaje, no un examen diagnóstico.

\section{Tiempo}

Como se mencionó anteriormente, generalmente se acepta que el examen se debe hacer entre la $14^{\mathrm{a}}$ y $20^{\mathrm{a}}$ semana de gestación. Varias publicaciones han demostrado que se puede hacer desde la semana 12 y luego de la semana 22.

Los datos normativos permiten interpretar con precisión exámenes antes de la semana 14 y luego de la semana 20 , pero la exactitud del examen a edades menores de 14 semanas y mayores de 20 semanas no está tan bien y tan ampliamente establecida como lo es en el período entre 14 y 20 semanas.

\section{BIBLIOGRAFIA}

1. Kass M., Shaw MW. «The Risk of Birth Defects: Jacobs vs. Theimer and the Parents Right to Know», Am. J. Law. \& Med. 1977; 2: 213245.

2. New. Engl. J. Med. 1991; 325: 6-10.

3. Canick JA., Knight GJ. «Multiple-marker screening for fetal Down syndrome», Contemporary Ob/Gyn 1992; 38: 25-42.
4. Crandall BF., Robinson L., Grau P. «Risks associated with an elevated maternal serum alphafetoprotein level, Am. J. Obstet. Gynecol. 1991; 165: 663-667.

5. Bogart M., Pandian MR., Jones W. «Abnormal chorionic maternal serum gonadotropin levels in pregnancies with fetal chromosome abnormalities», Prenatal Diag. 1987; 7: 623-630. 
6. Canick JA., Knight GJ., Palomaki GE et al. Low second-trimester maternal serum estriol in Down syndrome pregnancies. Am. J. Hum. Genet. 1987; 41: A269.

7. Knight GJ. «Techniques in Diagnostic Human Biochemical Genetics. A Laboratory Manual», Wiley-Liss, New York, 1991.

8. Drugan A et al. Similarity of twins to singleton MSAFP ratio by race: No need to establish specific multifetal tables. SPO Abstracts, No. 244, 1992.

9. Wald NJ., Cuckle HS et al. Maternal serum unconjugated oestriol and human chorionic gonadotropin levels in pregnancies with insulindependent diabetes: implications for screening for Down's syndrome. Br. J. Obstet. Gynecol. 1992; 99: 51-53.

10. Bogart MH. Prospective evaluation of maternal serum human chorionic gonadotropin levels in 3428 pregnancies. Am. J. Obstet. Gynecol. 1991; 165: 663-667.
11. Haddow JE., Palomaki GE., Knight GJ., Williams J., Pulkkinen A., Canick JA., Saller DN., Bowers GB. Prenatal screening for Down's syndrome with use of maternal serum markers. N. Engl. J. Med. 1992; 327: 588-593.

12. Heyl PS., Miller W., Canick JA. Maternal Serum Screening for Aneuploid Pregnancy by Alpha-Fetoprotein. hCG, and Unconjugated Estriol, Obstet. \& Gynecol. 1990; 76: 1025-1031.

13. Wald NJ., Cuckle HS., Densem JW et al. Maternal serum screening for Down's syndrome in early pregnancy. Br. Med. J. 1988; 297: 883 887.

14. Phillips OP., Elias $S$ et al. Maternal serum screening for fetal Down syndrome in women less than 35 years of age using alpha-fetoprotein, hCG and unconjugated Estriol: a prospective 2-year study, Obstet. \& Gynecol. 1992; 80: 353-358.

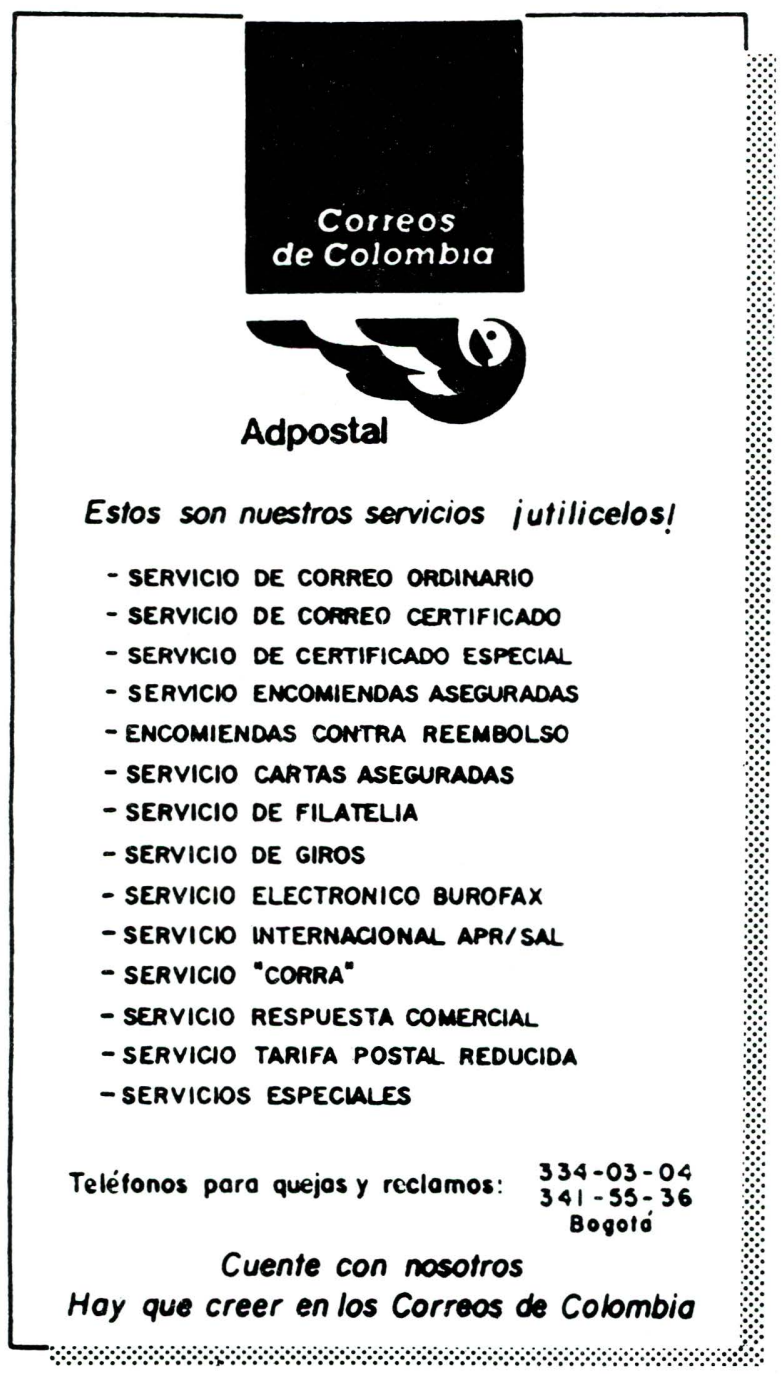

